\title{
Quantum Dot Light-Emitting Diode with Ligand-Exchanged $\mathrm{ZnCuInS}_{2}$ Quantum Dot
}

\author{
Takeshi FUKUDA $^{\dagger a)}$, Member, Masatomo HISHINUMA ${ }^{\dagger}$, Junya MAKI ${ }^{\dagger}$, and Hironao SASAKI ${ }^{\dagger}$, Nonmembers
}

\begin{abstract}
SUMMARY Nowadays, semiconductor quantum dots have attracted intense attention as emissive materials for light-emitting diodes, due to their high photoluminescence quantum yield and the controllability of their photoluminescence spectrum by changing the core diameter. In general, semiconductor quantum dots contain large amounts of organic ligands around the core/shell structure to obtain dispersibility in solution, which leads to solution processability of the semiconductor quantum dot. Furthermore, organic ligands, such as straight alkyl chains, are generally insulating materials, which affects the carrier transport in thin-film light-emitting diodes. However, a detailed investigation has not been performed yet. In this paper, we investigated the luminance characteristics of quantum-dot lightemitting diodes containing $\mathrm{ZnCuInS}_{2}$ quantum dots with different carbon chain lengths of alkyl thiol ligands as emitting layers. By evaluating the $\mathrm{CH}_{2} / \mathrm{CH}_{3}$ ratio from Fourier-transform infrared spectra and thermal analysis, it was found that approximately half of the oleylamine ligands were converted to alkyl thiol ligands, and the evaporation temperature increased with increasing carbon chain length of the alkyl thiol ligands based on thermogravimetric analysis. However, the photoluminescence quantum yield and the spectral shape were almost the same, even after the ligand-exchange process from the oleylamine ligand to the alkyl thiol ligand. The peak wavelength of the photoluminescence spectra and the photoluminescence quantum yield were approximately $610 \mathrm{~nm}$ and $10 \%$, respectively, for all samples. In addition, the surface morphology of spin coated $\mathrm{ZnCuInS}_{2}$ quantum-dot layers did not change after the ligand-exchange process, and the root-mean-square roughness was around $1 \mathrm{~nm}$. Finally, the luminance efficiency of an inverted device structure increased with decreasing carbon chain length of the alkyl thiol ligands, which were connected around the $\mathrm{ZnCuInS}_{2}$ quantum dots. The maximum luminance and current efficiency were $86 \mathrm{~cd} / \mathrm{m}^{2}$ and $0.083 \mathrm{~cd} / \mathrm{A}$, respectively.

key words: semiconductor quantum dot, quantum dot light-emitting diode, ligand exchange, alkyl thiol
\end{abstract}

\section{Introduction}

Organic light-emitting diodes (OLEDs) have been widely investigated for a long time, and flat-panel displays and general lighting employing OLEDs have already been implemented for practical use. By now, many kinds of organic emissive molecules, such as fluorescence, phosphorescence, and thermally activated delayed fluorescence materials, have been successfully synthesized for realizing high luminance efficiencies and long-term stability. On the other hand, semiconductor quantum dots (QDs) have also been used in emitting layers instead of the organic emissive molecules of OLEDs, and this type of device is known as a quantum-dot light-emitting diode (QLED) [1]. Semi-

Manuscript received February 23, 2017.

Manuscript revised April 25, 2017.

${ }^{\dagger}$ The authors are with the Department of Functional Materials Science, Saitama University, Suita-shi, 338-8570 Japan.

a)E-mail: fukuda@fms.saitama-u.ac.jp

DOI: $10.1587 /$ transele.E100.C.943 conductor QDs have several advantages compared with organic molecules, such as long-term stability [2], the possibility of solution processing, and controlled photoluminescence (PL) spectra by changing the core diameter [3]. By now, all-solution-processed devices [4], near-infrared emitters [5], and flexible device structures [6] have all been demonstrated with QLEDs.

In previous studies, Cd-based semiconductor QDs have been utilized as emissive materials for QLEDs due to their high PL quantum yield (PL-QY) and narrow PL spectra [7], [8]. However, Cd-based QDs are harmful to the environment and human health, and Cd-free semiconductor QDs have been strongly desired for a long time. InP [9], [10] and $\mathrm{CuInS}_{2}$ [11], [12] QDs were found to exhibit high PL-QYs and luminance performance for the corresponding QLEDs by optimizing synthesis conditions, such as the synthesis temperature, the reaction time, the concentration of raw materials, and the insertion of a carrier blocking layer. Previous papers demonstrated that PL-QYs of more than $80 \%$ were achieved, and this value is high enough for practical display and biological applications [13], [14]. Furthermore, the luminance performance of QLEDs with Cd-free QDs has been improved by controlling the organic ligand [15]; however, further investigation is required due to insufficient understanding of carrier dynamics in QLEDs.

Zn-doping techniques for $\mathrm{CuInS}_{2}$ and $\mathrm{AgInS}_{2}$ QDs have been widely investigated due to their blue-shifted PL wavelength and high PL-QY [16], [17]. Since the PL wavelength is determined by the core diameter and the band gap energy of bulk materials, the Zn-doping-induced PL wavelength shift is useful for realizing a precise control of the PL wavelength, which is strongly desired for display applications. However, high luminance efficiencies for QLEDs with $\mathrm{Zn}$-doped $\mathrm{CuInS}_{2}$ and $\mathrm{AgInS}_{2}$ have not been realized yet.

In this study, we investigated the optical and chemical properties of ligand-exchanged $\mathrm{ZnCuInS}_{2}$ QDs, in which the oleylamine ligands were exchanged with alkyl thiol ligands. We also evaluated QLEDs with $\mathrm{ZnCuInS}_{2}$ QD layers, and the carbon chain length of the alkyl thiol ligand was varied to investigate the relationship between the device performance and the length of the alkyl thiol ligand.

\section{Experimental}

A $\mathrm{ZnCuInS}_{2}$ QD solution in hexane was synthesized by a conventional hot soap method [18], [19]. At first, in- 
dium(III) acetate (312 mg, $1.07 \mathrm{mmol}$ ), copper(I) chloride (12.9 $\mathrm{mg}, 0.13 \mathrm{mmol})$, thiourea $(152 \mathrm{mg}, 2.0 \mathrm{mmol})$, zinc acetate dihydrate $(146.8 \mathrm{mg}, 0.67 \mathrm{mmol})$, and oleylamine $(40 \mathrm{ml})$ were mixed in a three-necked flask. After heating at $120{ }^{\circ} \mathrm{C}$ for $10 \mathrm{~min}$ to dissolve the aforementioned raw materials, the flask was heated at $220^{\circ} \mathrm{C}$ for $10 \mathrm{~min}$ in a nitrogen atmosphere, resulting in the formation of a $\mathrm{ZnCuInS}_{2}$ core. The synthesized QD solution was precipitated with excess acetone to remove unreacted raw materials. The solution was centrifuged at $20000 \mathrm{rpm}$ for $20 \mathrm{~min}$, and the supernatant was then decanted. The organic ligand of the QD was exchanged from oleylamine to alkyl thiol with a different carbon chain length through the above-mentioned procedure. Here octanethiol (sample A), 1-decanethiol (sample B), dodecanethiol (sample C), and hexadecanethiol (sample D) were used as the organic ligands. These alkyl thiol ligands were respectively injected in the synthesized $\mathrm{ZnCuInS}_{2}$ solution, and the mixed solutions were heated at $60^{\circ} \mathrm{C}$ for 4 $\mathrm{h}$ for the ligand-exchange process. Finally, residues were removed by a centrifugation process, and the concentration of the solution was controlled as $10 \mathrm{mg} / \mathrm{ml}$ for all the samples by evaporating hexane.

A typical device structure was glass substrate/indium tin oxide (ITO, $150 \mathrm{~nm}) / \mathrm{ZnO}(30 \mathrm{~nm}) / \mathrm{ZnCuInS}_{2} \mathrm{QDs} / 44^{\prime}{ }^{\prime}-$ bis(N-carbazolyl)-1,1'-biphenyl (CBP, $40 \mathrm{~nm}) / \mathrm{MoO}_{3}(10$ $\mathrm{nm}) / \mathrm{Al}(100 \mathrm{~nm})$ [19], which is generally known as an inverted structure [20], [21]. At first, a $\mathrm{ZnO}$ precursor solution containing zinc acetate dihydrate (precursor, $541 \mathrm{mg}$ ), 2-methoxyethanol (solvent, $5.0 \mathrm{ml}$ ), and monoethanolamine (stabilizer, $0.01 \mathrm{ml}$ ) was spin-coated on the top of an ITOcoated substrate, which was cleaned by ultrasonic wave and ultraviolet ozone treatments just before use. After spin coating the $\mathrm{ZnO}$ layer at a rotation speed of $3000 \mathrm{rpm}$, the sample was annealed at $220^{\circ} \mathrm{C}$ for $10 \mathrm{~min}$ [22]. A ZnCuInS 2 QD solution was subsequently spin coated at a rotation speed of $2000 \mathrm{rpm}$ for $1 \mathrm{~min}$, and the resulting device was annealed at $130{ }^{\circ} \mathrm{C}$ for $10 \mathrm{~min}$. Finally, $\mathrm{CBP}(40 \mathrm{~nm}), \mathrm{MoO}_{3}(10 \mathrm{~nm})$, and $\mathrm{Al}$ layers were thermally evaporated on the device in a vacuum chamber.

The PL spectrum was measured using a fluorescence meter (FluoroMax-3, Horiba Jobin Ivon). The Fouriertransform infrared (FT-IR) spectrum was measured using an FT-IR spectrometer (FT/IR-4700, JASCO). The PLQY was measured using a PL quantum yield measurement system (QEMS-2000, Systems Engineering). The thermal characteristics were measured using a thermo gravimetry-differential thermal analyzer (TG/DTA, Hitachi High-Technologies, TG-DTA6200). The surface morphology was evaluated using an atomic force microscope (AFM, Seiko, SPA-300). The current density-voltage, luminance-voltage, and current efficiency-current density characteristics were measured using a DC-voltage current source/monitor (6241A, ADCMT) and a luminance meter (LS-100, Konicaminolta).

\section{Results and Discussion}

Figure 1 shows the normalized PL spectra of $\mathrm{ZnCuInS}_{2}$ QD solutions dispersed in hexane, which contained different alkyl thiol ligands such as octanethiol (OT, sample A), 1-decanethiol (DT, sample B), dodecanethiol (DDT, sample C), and hexadecanethiol (HDT, sample D). The excitation wavelength was fixed at $375 \mathrm{~nm}$, and the carbon chain lengths of the alkyl thiol ligands were $8,10,12$, and 16 for OT, DT, DDT, and HDT, respectively. The PL spectra of all the $\mathrm{ZnCuInS}_{2}$ QDs with different alkyl thiol ligands showed almost the same peak wavelength at approximately $610 \mathrm{~nm}$, and the full width at half maximum (FWHM) was approximately $130 \mathrm{~nm}$ for all the samples. The FWHM of $\mathrm{CuInS}_{2}$ was much larger than that of CdSe/ZnS QDs (approximately 23-29 nm) [23], [24], and almost the same trend was observed even though $\mathrm{Zn}$ was doped in the $\mathrm{CuInS}_{2}$ QD. This is almost the same value as reported in previous papers [25]. In addition, PL-QYs of samples A, B, C, and D were determined to be $8.5,11.5,9.5$, and $9.6 \%$, respectively, by exciting ultraviolet light with the wavelength of $375 \mathrm{~nm}$. This result indicates that the carbon chain length of the connected alkyl thiol ligand did not affect the PL-QY of $\mathrm{ZnCuInS}_{2}$.

We then evaluated the chemical and physical characteristics of the ligand-exchanged $\mathrm{ZnCuInS}_{2}$ QD using TG/DTG analysis and FT-IR spectroscopy. Figures 2 (a) and 2 (b) show the FT-IR spectra of the raw materials used for the ligand-exchange process (i.e., octanethiol, 1decanethiol, dodecanethiol, and hexadecanethiol) and synthesized $\mathrm{ZnCuInS}_{2}$ QDs with different alkyl thiol ligands, respectively. These FT-IR spectra showed $\mathrm{CH}_{2}$ and $\mathrm{CH}_{3}$ bonds. Peaks at 2995 and $2863 \mathrm{~cm}^{-1}$ originate from the $\mathrm{CH}_{3}$ bond and those at 2922 and $2851 \mathrm{~cm}^{-1}$ correspond to the $\mathrm{CH}_{2}$ bond of the alkyl thiol ligand. This result indicates that the peaks from the $\mathrm{CH}_{2}$ bond increased with increasing

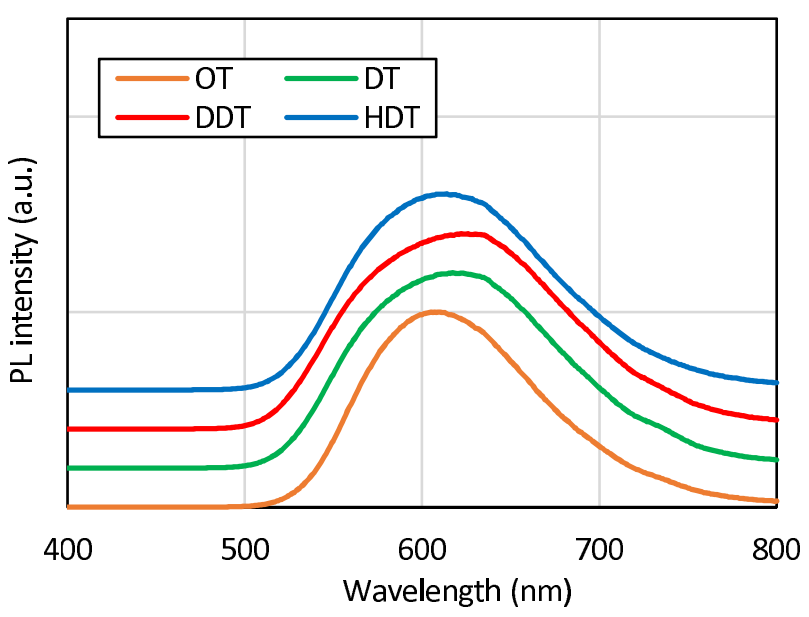

Fig. 1 PL spectra of $\mathrm{ZnCuInS}_{2}$ QDs with different alkyl thiol ligands. Here, octanethiol (OT, sample A), 1-decanethiol (DT, sample B), dodecanethiol (DDT, sample C), and hexadecanethiol (HDT, sample D) were used for the ligand-exchange process. The excitation wavelength was 375 nm. 


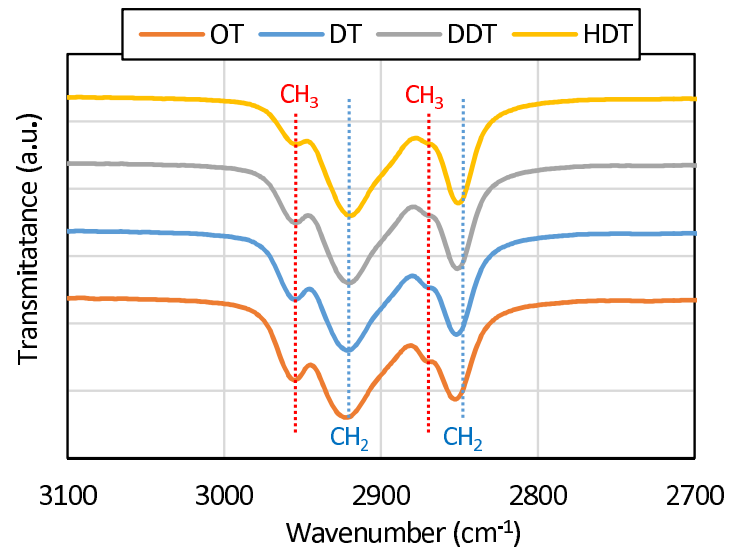

(a)

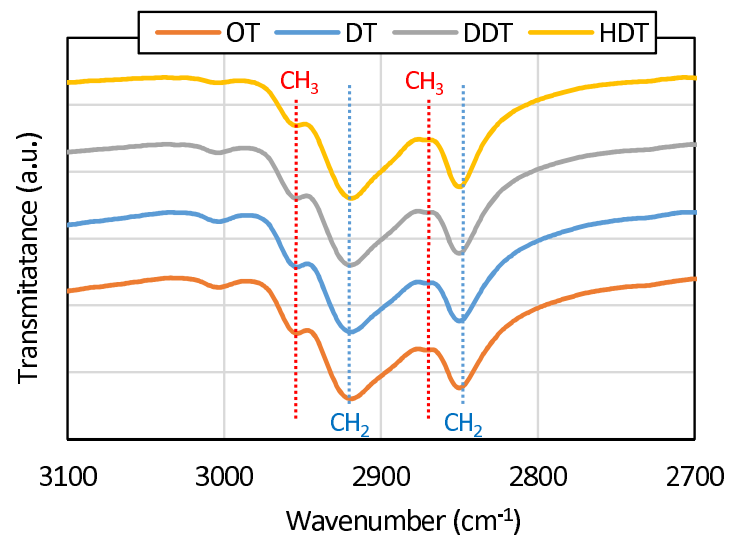

(b)

Fig. 2 FT-IR spectra of (a) alkyl thiol and (b) $\mathrm{ZnCuInS}_{2}$ QDs with different alkyl thiol ligands. The peaks at 2995 and $2863 \mathrm{~cm}^{-1}$ originate from the $\mathrm{CH}_{3}$ bond and those at 2922 and $2851 \mathrm{~cm}^{-1}$ correspond to the $\mathrm{CH}_{2}$ bond of the organic ligand.

carbon chain length. However, the FT-IR spectra of synthesized $\mathrm{ZnCuInS}_{2}$ QDs showed a broad absorbance ranging from 2800 to $3000 \mathrm{~cm}^{-1}$ as shown in Fig. 2 (b), which is due to the oleylamine solvent.

Next, we calculated the $\mathrm{CH}_{2} / \mathrm{CH}_{3}$ ratio from the FT-IR spectra in Fig. 2. Figure 3 shows the relationship between the carbon chain length of the alkyl thiol ligands and the $\mathrm{CH}_{2} / \mathrm{CH}_{3}$ ratio of raw materials and $\mathrm{ZnCuInS}{ }_{2}$ QDs. The $\mathrm{CH} 2 / \mathrm{CH} 3$ ratio continuously increased with increasing carbon chain length of the alkyl thiols for both cases. The reason for this is quite simple. One edge of the alkyl thiol contains the $\mathrm{CH} 3$ bond, and the rest part contains the $\mathrm{CH}_{2}$ bond. Therefore, the $\mathrm{CH}_{2} / \mathrm{CH}_{3}$ ratio corresponds to the carbon chain length of the alkyl thiol ligand as shown in Fig. 3, and the result in Fig. 3 (b) indicates that the oleylamine ligand of $\mathrm{ZnCuInS}_{2}$ QDs was successfully exchanged to the alkyl thiol ligand.

The thermal properties of $\mathrm{ZnCuInS}_{2}$ QDs were measured to investigate the weight ratio of the organic ligand to the $\mathrm{ZnCuInS}_{2} \mathrm{QD}$, and Tg of samples A-D are shown in Fig. 4 (a). Since all the alkyl thiol ligands used in this study evaporated below $300{ }^{\circ} \mathrm{C}$, the weight loss at $500{ }^{\circ} \mathrm{C}$ corre-

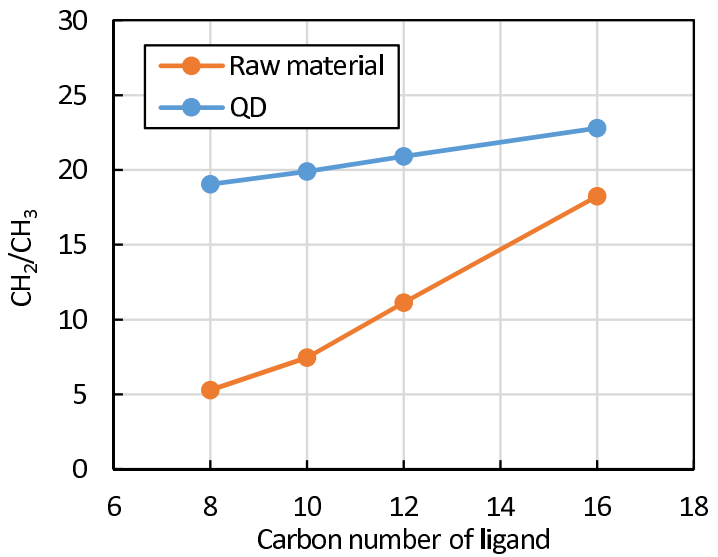

Fig. 3 Influence of the carbon chain length on the $\mathrm{CH}_{2} / \mathrm{CH}_{3}$ ratio of $\mathrm{ZnCuInS}_{2}$ QDs and alkyl thiols, which was calculated from the FT-IR spectra in Fig. 2.

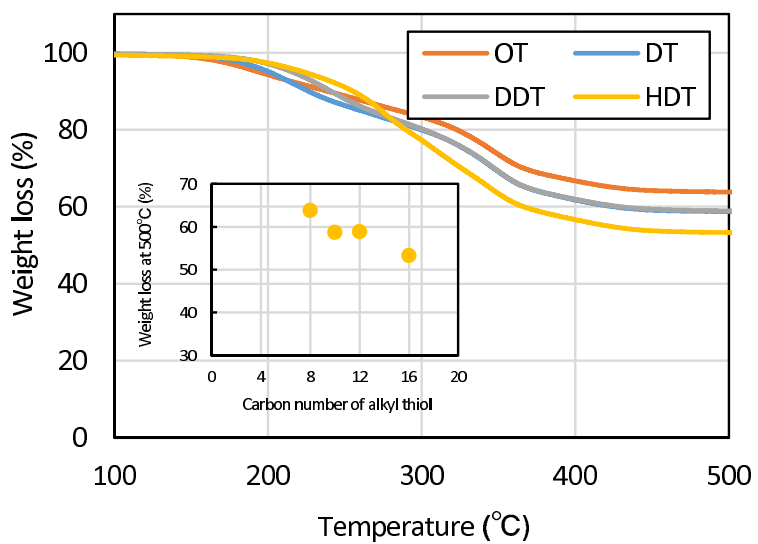

(a)

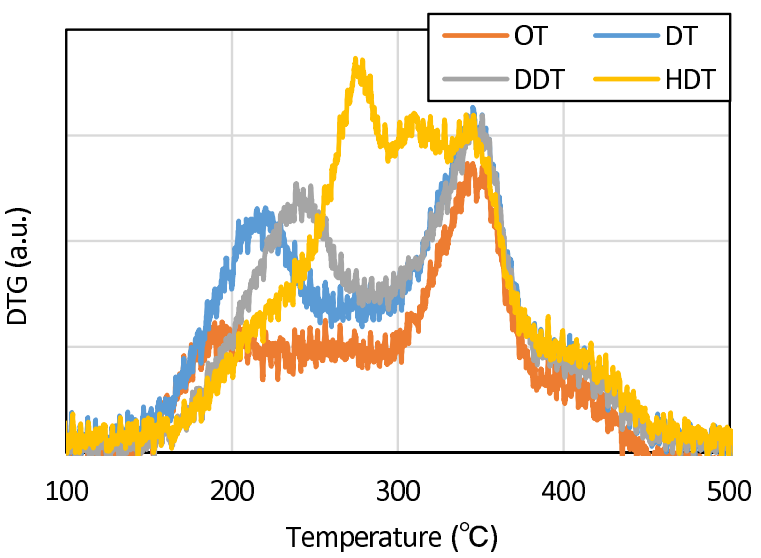

(b)

Fig. 4 (a) Tg and (b) DTG of $\mathrm{ZnCuInS}_{2}$ QDs with different alkyl thiol ligands. The inset of (a) shows the relationship between the carbon chain length of the alkyl thiol ligands and the weight loss at $500{ }^{\circ} \mathrm{C}$.

sponds to the amount of connected organic ligands around the $\mathrm{ZnCuInS}_{2}$ QD. As clearly shown in the inset of Fig. 4 (a), the weight loss at $500{ }^{\circ} \mathrm{C}$ decreased with decreasing carbon chain length of the alkyl thiol ligand. The molar ratio of oleylamine to alkyl thiol ligands can be calculated from the 


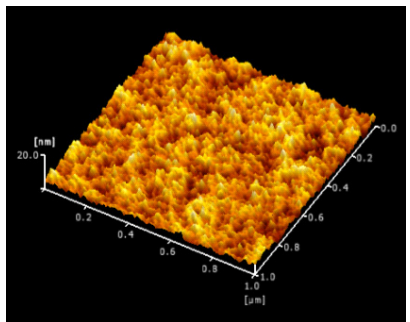

(a) OT

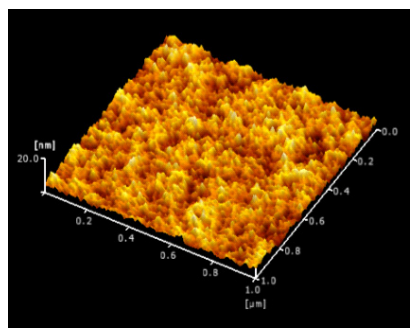

(c) DDT

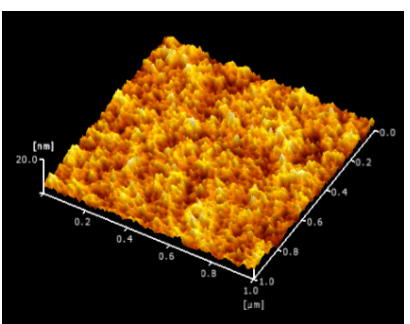

(b) DT

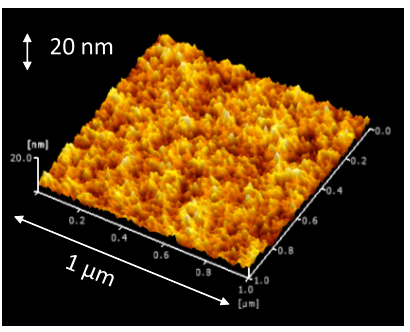

(d) HDT
Fig. 5 AFM images of spin coated $\mathrm{ZnCuInS}_{2}$ QD thin films, which were fabricated with different alkyl thiol ligands.

weight ratio and the molecular weights of oleylamine and alkyl thiol ligands; the molecular weights of octanethiol, 1decanethiol, dodecanethiol, and hexadecanethiol are 146.3, $174.0,202.4$, and 258.5 , respectively. As a result, the molar ratio of oleylamine to alkyl thiol ligands was 55:45 for all the samples, which was calculated from the $\mathrm{CH}_{2} / \mathrm{CH}_{3}$ ratio in Fig. 3; this result was caused by the difference in the molecular weights of the alkyl thiol ligands.

Figure 4 (b) shows the DTG of $\mathrm{ZnCuInS}_{2}$ QDs with alkyl thiol ligands containing different carbon chain lengths ranging from 8 to 16 . The feature at $340{ }^{\circ} \mathrm{C}$ in the DTG curve corresponds to the evaporation of oleylamine, which indicates that all the oleylamine ligands could not be exchanged with alkyl thiol ligands. This result is in good agreement with the FT-IR spectrum in Fig. 2 (b). In addition, the features at lower temperatures ranging from 220 ${ }^{\circ} \mathrm{C}$ to $280{ }^{\circ} \mathrm{C}$ correspond to the evaporation of alkyl thiol ligands, and the evaporation temperature was shifted toward the higher temperature region with increasing carbon chain length of the alkyl thiol ligands.

The surface morphology also affects the carrier injection from the carrier transport layer to the QD emitting layer in the QLED structure, and therefore, we evaluated the surface roughness of the spin coated $\mathrm{ZnCuInS}_{2}$ QD layer. The AFM images of the $\mathrm{ZnCuInS}_{2}$ QD thin films are shown in Fig. 5, and the alkyl thiol ligands used were (a) octanethiol, (b) 1-decanethiol, (c) dodecanethiol, and (d) hexadecanethiol. Aggregated QDs were not observed in the AFM images even when short alkyl thiol ligands were used. The root-mean-square roughnesses were 1.3, 1.2, 1.3, and $1.3 \mathrm{~nm}$ for samples A, B, C, and D, respectively. This result indicates that the carbon chain length of the alkyl thiol ligands did not affect the surface roughness of the QD layer, and these values are low enough to realize high luminance performance of thin-film emitting devices.

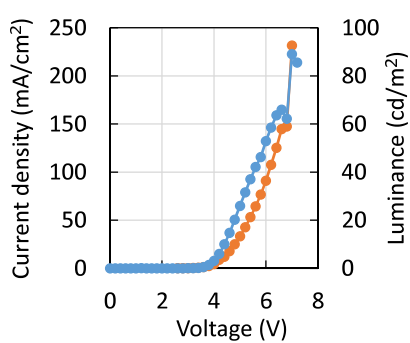

(a)

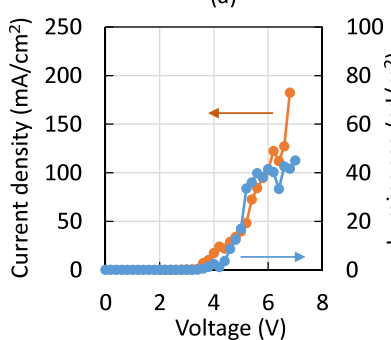

(c)

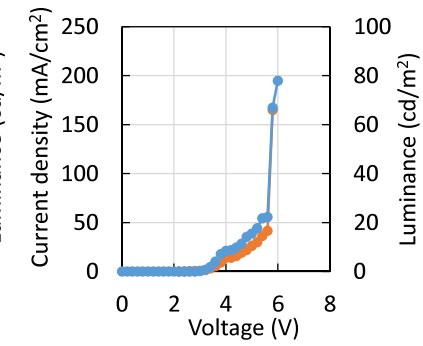

(b)

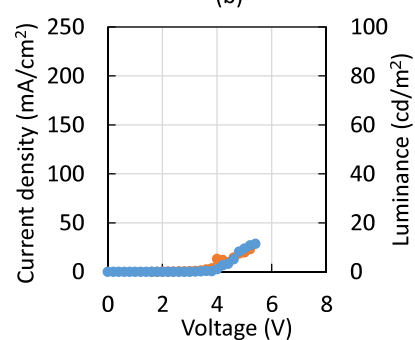

(d)
Fig. 6 Current density-voltage-luminance characteristics of QLEDs with $\mathrm{ZnCuInS}_{2}$ QDs with different carbon chain lengths of alkyl thiol ligands. The alkyl thiols used were (a) octanethiol, (b) 1-decanethiol, (c) dodecanethiol, and (d) hexadecanethiol.

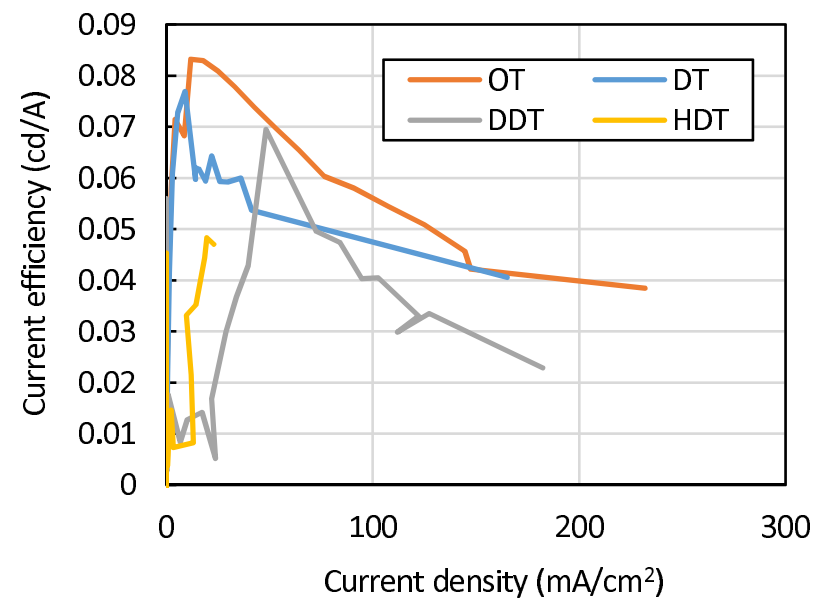

Fig. 7 Current efficiency-current density characteristics of QLEDs with $\mathrm{ZnCuInS}_{2}$ QDs with different alkyl thiol ligands.

Figure 6 shows the current density-voltage-luminance characteristics of QLEDs containing $\mathrm{ZnCuInS}_{2}$ QDs with different alkyl thiol ligands. Here, the alkyl thiols used were (a) octanethiol, (b) 1-decanethiol, (c) dodecanethiol, and (d) hexadecanethiol. A higher current density was observed when the alkyl thiol with a short carbon chain length was used for the ligand-exchange process. This result clearly indicates that long alkyl thiol ligands prevent carrier transport in the $\mathrm{ZnCuInS}_{2}$ layer, resulting in a low current density under the applied DC voltage. In addition, the luminance was also improved with decreasing carbon chain length of the alkyl thiol ligands due to efficient carrier transport. The highest luminance of $86 \mathrm{~cd} / \mathrm{m}^{2}$ was realized when octanethiol was used as the organic ligand.

We then estimated the current efficiencies of the de- 
vices. Figure 7 shows the current density-current efficiency characteristics, which were evaluated from the current density-voltage-luminance characteristics in Fig. 6. The current efficiency was improved with decreasing carbon chain length of the alkyl thiol ligands, and the highest current efficiency of $0.083 \mathrm{~cd} / \mathrm{A}$ was obtained. This value is quite low compared with reported QLEDs with Cd-free QDs such as InP and CuInS 2 [10], [15]. However, this is the first demonstration of QLEDs with ligand-exchanged $\mathrm{ZnCuInS}_{2}$ QDs, and the thick and multiple shell coating layers are useful techniques for realizing highly efficient QLEDs with $\mathrm{ZnCuInS}_{2}$ QDs.

\section{Conclusion}

In conclusion, we investigated the ligand-exchange process for $\mathrm{ZnCuInS}_{2}$ QDs and the relationship between the luminance performance and the carbon chain length of the alkyl thiol ligands, which was connected around the $\mathrm{ZnCuInS}_{2}$ QDs. By evaluating the FT-IR spectrum and Tg/DTG, it was found that approximately half of the oleylamine ligands were exchanged to alkyl thiol ligands with different carbon chain lengths. Finally, we successfully demonstrated efficient carrier transport and improved luminance when the alkyl thiol ligand with a short carbon chain length was used.

\section{References}

[1] K.-H. Lee, C.-Y. Han, H.-D. Kang, H. Ko, C. Lee, J. Lee, N.S. Myoung, S.-Y. Yim, and H. Yang, "Highly efficient, color-reproducible full-color electroluminescent devices based on red/green/blue quantum dot-mixed multilayer," ACS Nano, vol.9, no.11, pp.10941-10949, 2015.

[2] J. Pan, J. Chen, Q. Huang, Q. Khan, X. Liu, Z. Tao, W. Lei, F. Xu, and Z. Zhang, "Flexible quantum dot light-emitting diodes based on ZnO nanoparticles," RSC Adv., vol.5, no.100, pp.82192-82198, 2015.

[3] J.Y. Kim, O. Voznyy, D. Zhitomirsky, and E.H. Sargent, "25th anniversary article: Colloidal quantum dot materials and devices: A quarter-century of advances," Adv. Mater., vol.25, no.36, pp.4986-5010, 2013.

[4] X. Yang, Y. Ma, E. Mutlugun, Y. Zhao, K.S. Leck, S.T. Tan, H.V. Demir, Q. Zhang, H. Du, and X.W. Sun, "Stable, efficient, and all-solution-processed quantum dot light-emitting diodes with double-sided metal oxide nanoparticle charge transport layers," ACS Appl. Mater. Interfaces, vol.6, no.1, pp.495-499, 2014.

[5] X. Gong, Z. Yang, G. Walters, R. Comin, Z. Ning, E. Beauregard, V. Adinolfi, O. Voznyy, and E.H. Sargent, "Highly efficient quantum dot near-infrared light-emitting diodes," Nature Photonics, vol.10, no.4, pp.253-257, 2016.

[6] H.H. Kim, J.W. Shim, Y.-J. You, Y.J. Lee, C. Park, D.K. Hwang, and W.K. Choi, "Highly flexible inverted-quantum-dot lightemitting diodes on elastic polyurethane substrates," J. Mater. Chem. C, vol.5, no.7, pp.1596-1600, 2017. DOI: 10.1039/C6TC04310K.

[7] B.-H. Kang, J.-S. Lee, S.-W. Lee, S.-W. Kim, J.-W. Lee, S.-A. Gopalan, J.-S. Park, D.-H. Kwon, J.-H. Bae, H.-R. Kim, and S.-W. Kang, "Efficient exciton generation in atomic passivated CdSe/ZnS quantum dots light-emitting devices," Sci. Rep., vol.6, no.34659, 2016.

[8] X. Dai, Z. Zhang, Y. Jin, Y. Niu, H. Cao, X. Liang, L. Chen, J. Wang, and X. Peng, "Solution-processed, high-performance light-emitting diodes based on quantum dots," Nature, vol.515, no.7525, pp.96-99,
2014.

[9] J.-H. Jo, J.-H. Kim, K.-H. Lee, C.-Y. Han, E.-P. Jang, Y.R. Do, and $\mathrm{H}$. Yang, "High-efficiency red electroluminescent device based on multishelled InP quantum dots," Opt. Lett., vol.41, no.17, pp.3984-3987, 2016.

[10] J. Lim, M. Park, W.K. Bae, D. Lee, S. Lee, C. Lee, and K. Char, "Highly efficient cadmium-free quantum dot light-emitting diodes enabled by the direct formation of excitons within InP@ ZnSeS quantum dots," ACS Nano, vol.7, no.10, pp.9019-9026, 2013.

[11] J. Li, H. Jin, K. Wang, D. Xie, D. Xu, X. Xu, and G. Xu, "High luminance of $\mathrm{CuInS}_{2}$-based yellow quantum dot light emitting diodes fabricated by all-solution processing," RSC Adv., vol.6, no.76, pp.72462-72470, 2016.

[12] J.-H. Kim, K.-H. Lee, H.-D. Kang, B. Park, J.Y. Hwang, H.S. Jang, Y.R. Do, and H. Yang, "Fabrication of a white electroluminescent device based on bilayered yellow and blue quantum dots, Nanoscale," vol.7, no.12, pp.5363-5370, 2015.

[13] M.J. Anca, N.L. Pickett, N.C. Gresty, J.A. Harris, and K.C. Mishra, "Progress in non-Cd quantum dot development for lighting applications," ECS J. Solid State Sci. Technol., vol.2, no.2, pp.R3071-R3082, 2012.

[14] D. Deng, Y. Chen, J. Cao, J. Tian, Z. Qian, S. Achilefu, and Y. $\mathrm{Gu}$, "High-quality $\mathrm{CuInS}_{2} / \mathrm{ZnS}$ quantum dots for in vitro and in vivo bioimaging," Chem. Mater., vol.24, no.15, pp.3029-3037, 2012.

[15] Z. Bai, W. Ji, D. Han, L. Chen, B. Chen, H. Shen, B. Zou, and H. Zhong, "Hydroxyl-terminated $\mathrm{CuInS}_{2}$ based quantum dots: Toward efficient and bright light emitting diodes," Chem. Mater., vol.28, no.4, pp.1085-1091, 2016.

[16] D.K. Sharma, S. Hirata, L. Bujak, V. Biju, T. Kameyama, M. Kishi, T. Torimoto, and M. Vacha, "Influence of $\mathrm{Zn}$ on the photoluminescence of colloidal (AgIn) $\mathrm{Zn}_{2(1-x)} \mathrm{S}_{2}$ nanocrystals," Phys. Chem. Chem. Phys., vol.19, no.5, pp.3963-3969, 2017.

[17] H.C. Yoon, J.H. Oh, M. Ko, H. Yoo, and Y.R. Do, "Synthesis and characterization of green $\mathrm{Zn}-\mathrm{Ag}-\mathrm{In}-\mathrm{S}$ and red $\mathrm{Zn}-\mathrm{Cu}-\mathrm{In}-\mathrm{S}$ quantum dots for ultrahigh color quality of down-converted white LEDs," ACS Appl. Mater. Interfaces, vol.7, no.13, pp.7342-7350, 2015.

[18] J. Kwak, W.K. Bae, D. Lee, I. Park, J. Lim, M. Park, H. Cho, H. Woo, D.Y. Yoon, K. Char, S. Lee, and C. Lee, "Bright and efficient full-color colloidal quantum dot light-emitting diodes using an inverted device structure," Nano Lett., vol.12, no.5, pp.2362-2366, 2012.

[19] M. Hishinuma, T. Fukuda, J. Maki, N. Kamata, and Z. Honda, "Quantum dot light-emitting diode with ligand-controlled $\mathrm{CuInS}_{2} / \mathrm{ZnS}$ quantum dot," Proc. 23rd international display workshops with conjunction with Asia display 2016, pp.763-766, 2016.

[20] H. Zhang, H. Li, X. Sun, and S. Chen, "Inverted quantum-dot light-emitting diodes fabricated by all-solution processing," ACS Appl. Mater. Interfaces, vol.8, no.8, pp.5493-5498, 2016.

[21] H.-M. Kim, J.-H. Youn, G.-J. Seo, and J. Jang, "Inverted quantumdot light-emitting diodes with solution-processed aluminiumzinc oxide as a cathode buffer," J. Mater. Chem. C, vol.1, no.8, pp.1567-1573, 2013.

[22] Y. Sun, J.H. Seo, C.J. Takacs, J. Seifter, and A.J. Heeger, "Inverted polymer solar cells integrated with a low temperatureannealed sol-gel derived $\mathrm{ZnO}$ film as an electron transporting layer," Adv. Mater., vol.23, no.14, pp.11679-1683, 2011.

[23] S.-C. Shei, W.-J. Chiang, and S.-J. Chang, "Synthesis of CuInS 2 quantum dots using polyetheramine as solvent," Nanoscale Res. Lett., vol.10, no.1, p.122, 2015.

[24] P. Yang, M. Ando, T. Taguchi, and N. Murase, "Highly luminescent $\mathrm{CdSe} / \mathrm{Cd}_{x} \mathrm{Zn}_{1-x} \mathrm{~S}$ quantum dots with narrow spectrum and widely tunable wavelength," J. Phys. Chem. C, vol.115, no.30, pp.14455-15560, 2011.

[25] W. Chung, H. Jung, C.H. Lee, and S.H. Kim, "Fabrication of highly stable silica coated $\mathrm{ZnCuInS}$ nanocrystals monolayer via layer by layer deposition for LED application," Opt. Express., vol.22, no.3, pp.2483-2499, 2014. 


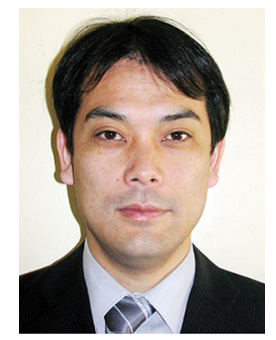

Takeshi Fukuda received B.E. and M.E. degrees from Waseda University, Tokyo, Japan, in 1999 and 2001, respectively. In 2001, he joined Fujikura Ltd. He received Ph.D. degree from Shinshu University, Nagano, Japan, 2008. Since 2008, he has been the assistant professor with the department of functional materials science, Saitama University, Saitama, Japan.

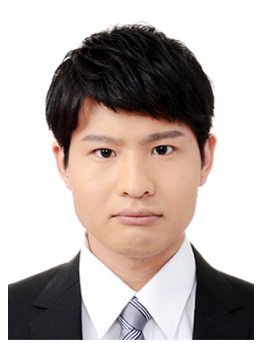

Masatomo Hishinuma

received B.E. degree from Saitama University, Saitama, Japan, in 2015. Now, he is the master course student with the graduate school of science and engineering, Saitama University, Saitama, Japan.

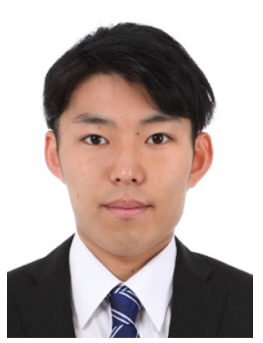

Junya Maki received B.E. degree from Saitama University, Saitama, Japan, in 2016. Now, he is the master course student with the graduate school of science and engineering, Saitama University, Saitama, Japan.

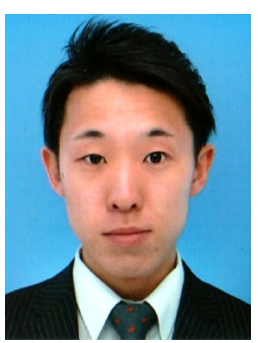

Hironao Sasaki is the bachelor course student with the department of functional materials science, Saitama University, Saitama, Japan. 